\title{
Cosmic rays from Galactic pulsars
}

\author{
W. Bednarek and M. Bartosik
}

\author{
Department of Experimental Physics, University of Łódź, ul. Pomorska 149/153, 90-236 Łódź, Poland \\ e-mail: bednar@fizwe4.fic.uni.lodz.pl
}

Received 5 January 2004 / Accepted 11 May 2004

\begin{abstract}
We calculate energy spectra and mass composition of cosmic rays accelerated by the galactic population of pulsars during their radio and gamma-ray phase. It is assumed that a significant part of the pulsar rotational energy is lost on acceleration of iron nuclei extracted from the surface of the neutron star. The nuclei are accelerated, at first when passing the outer gap of the inner pulsar magnetosphere and later in the pulsar wind zone, to energies corresponding to $50 \%$ of the total potential drop through the polar cap region of the neutron star. We calculate energy spectra of the nuclei injected from the pulsar wind nebulae into the Galaxy including different energy loss processes of nuclei during their propagation in the pulsar magnetosphere and the expanding nebula, their fragmentation in collisions with radiation and matter, adiabatic energy losses, and escape from the nebula. Several models proposed for the distribution of the initial parameters of the galactic pulsar population are considered. It is shown that the best description of the observed cosmic ray spectrum and the mass composition between a few $10^{15} \mathrm{eV}$ and a few $10^{18} \mathrm{eV}$ is obtained for the model $\mathrm{B}$ of Lorimer et al., in which the logs of initial pulsar periods and surface magnetic fields are given by the Gaussian distributions with the average values of $\langle\log P[\mathrm{~ms}]\rangle=2.6$ and $\langle\log B[G]\rangle=12.3$, respectively. In order to supply the cosmic rays into the Galaxy with the required rate, the product of the efficiency of cosmic ray acceleration by pulsars and their birth rate should be about $10^{-2} \mathrm{yr}^{-1}$.
\end{abstract}

Key words. stars: supernovae: general - acceleration of paricles - stars: pulsars: general - ISM: cosmic rays

\section{Introduction}

The origin of cosmic rays (CRs) with energies around and above $\sim 10^{15} \mathrm{eV}$, the knee region, is still far from being understood. It is widely accepted that the lower energy CRs, below the knee, are accelerated at large scale shock waves created in the interstellar medium by supernova explosions. It is argued that in some favorite conditions the particles might be also accelerated above $10^{15} \mathrm{eV}$ by this same supernova shock mechanism if, e.g. multiple supernovae exploded in the star forming regions - superbubbles (Cesarsky \& Montmerle 1983; Bykov 2001), a supernova explodes in its dense wind (Biermann 1993; Stanev et al. 1993), the magnetic field at the shock front is non-linearly amplified by CRs (Lucek \& Bell 2000; Bell \& Lucek 2001; Drury et al. 2003), or superheavy nuclei are accelerated (Hörandel 2003). The acceleration of particles to such energies might also occur in a large scale terminal shock of the galactic wind (Jokipii \& Morfill 1985). Other models propose that the observed cosmic rays has extragalactic origin. For example, Protheroe \& Szabo (1992) consider their acceleration in the shocks in the accretion flows in active galactic nuclei, and Vietri (1995) and Waxman (1995) argue for acceleration on relativistic shocks of gamma-ray bursts (GRBs). This last idea has been considered recently in a more detail by Wick et al. (2004), who propose the origin of cosmic rays from $\sim 10^{14} \mathrm{eV}$ up to the highest energies in the GRBs including the events which occurred in our own Galaxy with typical rate of $(3-10) \times 10^{-6}$ per yr. For the extensive review of recent models of the cosmic ray acceleration we refer to Hörandel (2004).

Another acceleration mechanism was proposed long time ago, soon after the pulsar discovery. It postulated acceleration of CRs above the knee region in the pulsar wind zone in large amplitude electromagnetic waves generated by rotating neutron star (Ostriker \& Gunn 1969; Karakuła et al. 1974). The contribution of particles accelerated by pulsars to the observed cosmic ray spectrum has been more recently discussed in detail by Cheng \& Chi (1996), Bednarek \& Protheroe (2002), Giller \& Lipski (2002). For example, Bednarek \& Protheroe (2002) estimate the contribution of heavy nuclei accelerated in the pulsar outer gaps (Cheng et al. 1986) to energies above the knee region. Giller \& Lipski (2002) derive the initial parameters of the pulsar population inside the Galaxy required to explain the observed shape of the CR spectrum and its intensity. Note that also CRs with extremely high energies, i.e. above the ankle at $\sim 10^{18} \mathrm{eV}$, might be accelerated in the wind regions of pulsars with super strong surface magnetic fields, objects called magnetars (Blasi et al. 2000; Arons 2003).

The models postulating acceleration of CRs between the knee and the ankle locally in our Galaxy obtained strong support by recent observation of the cosmic ray anisotropies from the direction of the Galactic Centre and the Cygnus region (Hayashida et al. 1999; Bellido et al. 2001). The AGASA 
experiment (Hayashida et al. 1999) reported the excesses of CRs in the narrow energy range close to $\sim 10^{18} \mathrm{eV}$ with the significance of $4.5 \sigma$ and $3.9 \sigma$ for the Galactic Centre and the Cygnus region, respectively.

Another information on the origin of CRs can be reached by measuring its mass composition. Most of the experiments suggest that the average mass composition of CRs is relatively light below the knee (e.g., Burnett et al. 1990; Apanasenko et al. 2001) and becomes heavier above the knee (e.g., Glasmacher et al. 1999; Arqueros et al. 2000; Fowler et al. 2001; Shirasaki et al. 2001; Ave et al. 2003). Recent preliminary results obtained from the analysis of the KASCADE data confirm heavier composition above the knee and show evidences of the rigidity dependent break in the spectra of specific groups of nuclei (e.g., Roth et al. 2003). The mass composition seems to decline again to lighter between $10^{17}-10^{18} \mathrm{eV}$, i.e. at the region of the second knee, according to some experiments (Bird et al. 1993; Yoshida et al. 1995; Dawson et al. 1998), or may be still quite heavy in this energy range as it is obtained in the analysis of the Haverah Park data which show bi-modal composition composed of one third of protons and two thirds of iron (Abu-Zayyad et al. 2001). Recent analysis of the Vulcano Range data also suggest heavy composition composed of $88 \%$ of the iron nuclei in the bi-modal proton-iron composition at a median energy of $10^{18} \mathrm{eV}$ (Dova et al. 2003). The change of the cosmic ray mass composition at the knee region is often explained as a result of rigidity dependent acceleration causing heavy nuclei start to dominate at higher energies (Peters 1961). The heavy nuclei enrichment of the galactic CRs might be also due to propagation effects in the galactic disk and halo (e.g., Maurin et al. 2003). Moreover, a contribution of another type of sources to CRs above the knee region, e.g. pulsars, may also increase its average mass.

In this paper we consider the last possibility in more details. We calculate the energy spectra of different types of nuclei injected by the pulsars into the Galaxy, applying a model for their injection and propagation in the expanding pulsar wind nebula (PWNa) surrounding a young pulsar. The model takes into account the energy losses and escape conditions of nuclei during the expansion of the nebula. Similar model has been recently applied for modelling the $\gamma$-ray emission from the PWNe (Bednarek \& Bartosik 2003). We estimate the contribution of nuclei, escaping from the PWNe, to the observed cosmic ray spectrum above the knee for different models of the pulsar population in the Galaxy. The results of calculations are compared with the reports on the mass composition in this energy region.

\section{The energy spectra of nuclei injected from the pulsar wind nebulae}

In this section we calculate spectra of nuclei accelerated by young pulsars and injected from the pulsar wind nebulae into the Galactic medium. We define the acceleration mechanism of nuclei, consider the effects of their propagation in the expanding nebula, and discuss the results for different models of the galactic pulsar population.

\subsection{Acceleration of nuclei by the pulsar}

It is likely that rotating magnetospheres of neutron stars can accelerate not only leptons but also heavy nuclei, extracted from positively charged polar cap regions. In fact different aspects of high energy phenomena around pulsars may need the presence of heavy nuclei. For example, a flow of iron nuclei, coexisting with an outflowing electron-positron plasma, is postulated in order to explain the change in the drift direction of the radio subpulses (Gil et al. 2003). The presence of heavy nuclei can also explain morphological features of the Crab Nebula and the appearance of extremely energetic leptons inside the nebula, accelerated as a result of resonant scattering of positrons and electrons by heavy nuclei (Hoshino et al. 1992; Gallant \& Arons 1994). From normalization to the observations of the Crab pulsar, Arons and collaborators (e.g., see, Arons 1998) postulate that the Lorentz factors of iron nuclei, accelerated somewhere in the inner magnetosphere and the pulsar wind zone and, injected into the pulsar wind nebula should be,

$\gamma_{\mathrm{Fe}} \approx \chi Z e \Phi_{\mathrm{open}} / m_{\mathrm{i}} c^{2} \approx 8 \times 10^{9} \chi B_{12} P_{\mathrm{ms}}^{-2}$,

where $m_{\mathrm{i}}$ and $Z e$ are the mass and charge of the iron nuclei, $c$ is the velocity of light, and $\Phi_{\text {open }}=\sqrt{L_{\text {rot }} / c}$ is the total electric potential drop across the open magnetosphere, $L_{\text {rot }}$ is the rate of rotational energy lost by the pulsar, $B=10^{12} B_{12} G$ is the surface magnetic field of the pulsar, and $P=10^{-3} P_{\mathrm{ms}}$ is the pulsar period. Due to the rotational energy losses on emission of dipole radiation, the pulsar period evolves in time according to

$P_{\mathrm{ms}}^{2}=P_{0, \mathrm{~ms}}^{2}+2 \times 10^{-9} t B_{12}^{2}$,

where $P_{0, \mathrm{~ms}}$ is the initial period of the pulsar, and $t$ is in seconds. Arons and collaborators argue that the acceleration factor $\chi$ is not far from unity. $\chi=0.5$ is taken in the following calculations. Moreover, we assume that these nuclei take significant part, $\xi$, of the pulsar rotational energy. The unknown value of $\xi$ times the pulsar birth rate in the Galaxy, $\eta$, can be obtained from the comparison of contribution of particles injected by pulsars to the observed cosmic ray flux. Equation (1) postulates that the pulsar at a specific time accelerates nuclei monoenergetically. However the energies of freshly produced nuclei change in time due to the change of the pulsar period caused by its rotational energy losses.

We assume that a pulsar can extract iron nuclei from its surface and accelerate them to high energies during the radio phase. Then the products of efficient leptonic cascades, occurring in the inner pulsar magnetosphere, impinge on the polar cap region allowing extraction of the iron nuclei from the surface.

The iron nuclei extracted from the surface of the pulsar are accelerated in the outer gaps of the inner pulsar magnetosphere (Cheng et al. 1986), suffering partial photo-disintegrations in collisions with nonthermal radiation produced in leptonic cascades. The details of the acceleration model of nuclei in the outer gaps are given in Sect. 3.1 of Bednarek \& Protheroe (2002). The level of photo-disintegration of nuclei depends on the pulsar parameters. To calculate the number of nucleons extracted from nuclei during propagation through the outer gap we determine the outer gap radiation field 
(Bednarek \& Protheroe 2002), by interpolation/extrapolation of the radiation fields in the Crab type pulsars, calculated by Ho (1989), and for the Vela type pulsars, based on the observed change of the $\gamma$-ray luminosity (Ruderman \& Cheng 1988). Nuclei and protons from their disintegration are additionally accelerated in the pulsar wind zone to energies postulated by Arons (1998), see Eq. (1). They are injected into the pulsar wind nebula. Neutrons from the disintegration of nuclei move ballistically through the nebula decaying inside or outside it depending on their Lorentz factor. At the early stage of expansion, when the nebula is dense enough, nuclei suffer further disintegrations in collisions with the matter (Bednarek \& Protheroe 2002). These processes are discussed in the next subsection.

\subsection{Escape of nuclei from expanding nebula}

The nuclei, injected by the pulsar, propagate in the expanding nebula with parameters changing drastically in time. In order to take properly into account different effects on their propagation (collisions with matter, diffusion inside the nebula, escape from the nebula), we have to assume a time dependent model for the expanding nebula taking into account not only the initial parameters of the expanding envelope but also the energy supplied by the pulsar. The model has to determine such basic parameters as: the expansion velocity of the nebula, the pulsar wind shock radius, the outer radius of the nebula, the average density of matter and magnetic field strength inside the nebula, and others. The details of such a simple model for the PWNe are described in our previous paper (Bednarek \& Bartosik 2003). We repeat here its main features.

The evolution of a supernova remnant, containing an energetic pulsar, is described according to the picture considered by Ostriker \& Gunn (1971) and Rees \& Gunn (1974). Let us denote the initial expansion velocity of the bulk matter in supernova envelope by $V_{0, \mathrm{SN}}$ and its initial mass by $M_{0, \mathrm{SN}}$. The expansion velocity can increase due to the additional supply of energy to the nebula by the pulsar. It can also decrease due to the accumulation of the surrounding matter. We take these processes into account to determine the radius of the nebula at a specific time, $t$, by using the energy conservation,

$$
\frac{M_{\mathrm{SN}}(t) V_{\mathrm{SN}}^{2}(t)}{2}=\frac{M_{0, \mathrm{SN}} V_{0, \mathrm{SN}}^{2}}{2}+L_{\mathrm{pul}-\mathrm{neb}}
$$

where $L_{\text {pul-neb }}$ is the part of the energy supplied by the pulsar to the nebula in the form of magnetic field and the energy transfered from relativistic hadrons to the nebula due to adiabatic energy losses. The rest of the rotational energy lost by the pulsar is almost immediately radiated by accelerated leptons. The energy of relativistic hadrons which is injected into the interstellar medium from the nebula is equal to the difference between the energy gained by hadrons during acceleration process, assumed equal to $\xi L_{\text {rot }}$, and the energy lost by them to the nebula during its adiabatic expansion. The part of energy lost by relativistic hadrons during adiabatic expansion of the nebula has been calculated numerically since the process of diffusion of hadrons inside the nebula has to be taken into account.
The mass of the expanding nebula increases (by sweeping the surrounding medium) according to the formula

$M_{\mathrm{SN}}(t)=M_{0, \mathrm{SN}}+\frac{4}{3} \pi \rho_{\mathrm{sur}} R_{\mathrm{Neb}}^{3}(t)$,

where $\rho_{\text {sur }}$ is the density of the surrounding medium and $R_{\mathrm{Neb}}$ is the outer radius of the expanding envelope at the time, $t$, which depends on the expansion history of the nebula,

$R_{\mathrm{Neb}}=\int_{0}^{t} V_{\mathrm{SN}}\left(t^{\prime}\right) \mathrm{d} t^{\prime}$

The expansion velocity of the nebula, $V_{\mathrm{SN}}(t)$, and the average density of matter inside it,

$\rho_{\mathrm{Neb}}=3 M_{\mathrm{SN}}(t) / 4 \pi R_{\mathrm{Neb}}^{3}(t)$,

at an arbitrary time have been found by solving numerically the above set of Eqs. (3)-(6). Note however, that the average density of matter, which is very useful for our simple model, may not correspond to the real distribution of matter inside the nebula specially at the later phase of expansion when the part of matter can form dense filaments. For example the matter inside the Crab Nebula is accumulated in filaments containing $4.6 \pm$ $1.8 M_{\odot}$ of ionized and neutral gas (Fesen et al. 1997) with density $\sim 500 \mathrm{~cm}^{-3}$ (Davidson \& Fesen 1985). No significant gas has been confirmed around the Crab Nebula (Fesen et al. 1997). Atoyan \& Aharonian (1996) suggest that relativistic hadrons can be efficiently trapped by these dense filaments. If this is the case then the efficiency of interactions of relativistic nuclei with the matter can be enhanced in respect to the interactions with the uniformly distributed matter inside the nebula.

The pulsar loses energy in the form of a relativistic wind extending up to the shock at a distance $R_{\mathrm{sh}}$. At this distance, the pressure of the wind is balanced by the pressure of the expanding nebula. Rees \& Gunn (1974) estimate the location of this shock as a function of time by comparing the pulsar wind energy flux, determined by $L_{\text {rot }}$ (see Eq. (8)), with the pressure of the outer nebula, determined by the supply of energy to the nebula by the pulsar over the whole of its lifetime,

$\frac{L_{\mathrm{rot}}(t)}{4 \pi R_{\mathrm{sh}}^{2} c} \approx \frac{L_{\mathrm{pul}-\mathrm{neb}}}{\frac{4}{3} \pi R_{\mathrm{Neb}}^{3}}$

and

$L_{\mathrm{rot}}(t)=B_{\mathrm{s}}^{2} R_{\mathrm{s}}^{6} \Omega^{4} / 6 c^{3} \approx 2.5 \times 10^{43} B_{12}^{2} P_{\mathrm{ms}}^{-4} \operatorname{erg~s}^{-1}$,

where $R_{\mathrm{S}}$ and $B_{\mathrm{S}}$ are the radius of the pulsar and its surface magnetic field, $\Omega=2 \pi / P$. The pulsar period changes according to Eq. (2). It is assumed in this formula that $R_{\mathrm{sh}} \ll R_{\mathrm{Neb}}$.

Knowing how the magnetic field depends on the distance from the pulsar in the pulsar wind zone, we can estimate the strength of the magnetic field at the shock region from

$B_{\mathrm{sh}}=\sqrt{\sigma} B_{\mathrm{s}}\left(\frac{R_{\mathrm{s}}}{R_{\mathrm{lc}}}\right)^{3} \frac{R_{\mathrm{lc}}}{R_{\mathrm{sh}}}$,

where $\sigma$ is the ratio of the magnetic energy flux to the particle energy flux from the pulsar at the location of the pulsar wind shock. There is observational evidence that $\sigma$ evolves in time. It is much less than one for very young nebulae (of the 
Crab type), and increases with age being of the order of one for nebulae of the Vela type. The value of $\sigma$ for a specific pulsar is determined by the efficiency of conversion of magnetic energy into the particles energy in the pulsar wind zone. The evolution of $\sigma$ with the parameters of the pulsar is estimated by interpolating between the values for the Crab pulsar, equal to $\sim 0.003$ (Kennel \& Coroniti 1984), and for the Vela pulsar, equal to $\sim 1$ (Helfand et al. 2001). We assume that the magnetic field strength in the volume of the nebula depends on the distance from its center as described by the formulae derived in the magnetohydrodynamic model for the PWNe considered by Kennel \& Coroniti (1984). The geometry of the magnetic field inside the nebula may have strong effect on the diffusion of nuclei inside it. Recent 3-dimensional modelling of the Chandra observations of the Crab Nebula suggests that the regular component of the magnetic field should be comparable to the turbulent component (Shibata et al. 2003). In our calculations the diffusion in turbulent component is only considered. The presence of regular toroidal magnetic field should slow down the diffusion of nuclei through the nebula.

It is assumed that nuclei injected into the nebula at a specific time $t_{\text {inj }}$, escape from it at the time $t_{\mathrm{esc}}$, if their diffusion distance in the turbulent magnetic field of the nebula, $R_{\text {diff }}$, is equal to the dimension of the nebula, $R_{\mathrm{Neb}}$, at the time $t_{\mathrm{esc}}$. The diffusion distance in the magnetic field of the nebula is obtained by integration (Bednarek \& Protheroe 2002),

$R_{\mathrm{diff}}=\int_{t_{\text {inj }}}^{t_{\mathrm{esc}}} \sqrt{\frac{3 D}{2 t^{\prime}}} \mathrm{d} t^{\prime}$,

where the diffusion coefficient is taken to be $D=R_{\mathrm{L}} c / 3$, and $R_{\mathrm{L}}$ is the Larmor radius of nuclei depending on the distance from the center of the nebula. $R_{\text {diff }}$ and $t_{\text {esc }}$ have been calculated numerically. We include also the adiabatic losses of nuclei during the period of their propagation inside the nebula. Due to this losses and interaction of nuclei with the matter the energy of a nucleus at a specific time is

$E(t)=E\left(t_{\mathrm{inj}}\right) \frac{t_{\mathrm{inj}}+t}{2 t_{\mathrm{inj}} K^{\tau}}$

where $E\left(t_{\text {inj }}\right)$ is its energy at the time $t_{\text {inj }}, K$ is the inelasticity coefficient for collisions of nuclei with the matter, and $\tau$ is the optical depth calculated based on the known density of matter inside the nebula (see Eq. (7)). The details of these calculations are given in Sects. 4.2 and 4.3 in Bednarek \& Protheroe (2002).

Considering all the processes discussed above, we calculate the energy spectra of different groups of nuclei escaping from the nebula into the Galaxy for arbitrary initial parameters of the pulsars. Protons from decay of neutrons, released from heavy nuclei during their propagation through the radiation field of the outer gap of the pulsar and later as a result of collisions with the matter inside the nebula, are also included in these calculations. Neutrons extracted from nuclei in collisions with the outer gap radiation field have relatively low Lorentz factors since their parent nuclei are pre-accelerated in the outer gap to energies lower than that achieved later in the pulsar wind zone. On the other hand, neutrons extracted from nuclei in collisions with the matter have typically higher Lorentz factors than protons since, moving balistically through the PWNa, do not lose energy on the adiabatic expansion. We assume that neutrons decaying within $3 \mathrm{kpc}$ from the galactic disk are captured by the magnetic field of the galactic halo and contribute to the galactic CRs. As an example, Figs. 1 show the spectra of different types of nuclei injected into the Galaxy for the case of a supernova with initial parameters derived for the Crab Nebula, i.e. the mass of the supernova envelope equal to $4 M_{\odot}$, the initial expansion velocity $2000 \mathrm{~km} \mathrm{~s}^{-1}$, and the density of surrounding medium equal to $0.3 \mathrm{~cm}^{-3}$. Figures $1 \mathrm{a}-\mathrm{c}$ show spectra of the escaping nuclei within selected range of mass numbers for pulsars with initial periods $1 \mathrm{~ms}, 10 \mathrm{~ms}$, and $100 \mathrm{~ms}$, and a typical surface magnetic field of $4 \times 10^{12} \mathrm{G}$. It is clear that nuclei injected by pulsars with shorter initial periods suffer more severe fragmentations. The total spectra of these secondary nuclei can be even dominated in some range of energies by secondary protons extracted from heavy nuclei and from decay of neutrons (thin full curves). The total spectra of the nuclei above $\sim 10^{(7-8)} \mathrm{GeV}$ have the spectral index not far from -2 extending through about two decades in energy. The low energy bump in the spectra below $\sim 10^{6} \mathrm{GeV}$ is due to the neutrons extracted from nuclei during their fragmentation in the radiation field of the outer gap of the pulsar. Figure 1d compares the total spectra of nuclei injected by pulsars with different initial periods and initial expansion velocities of the supernova envelopes. Pulsars with longer initial periods should be able to contribute to lower energy range in the total spectrum of nuclei. On the other hand, larger initial expansion velocity of the envelope allows the nuclei to escape at earlier times because the magnetic field inside the envelope drops more rapidly. Therefore, these nuclei escape with larger energies due to their smaller energy losses on the adiabatic expansion.

\subsection{Pulsar population within the Galaxy}

The spectrum of nuclei injected inside the Galaxy can be obtained by summing up over all population of pulsars inside the Galaxy. However, the initial parameters of the pulsars are not precisely known. Therefore, we consider a few different models proposed in the literature. They differ in distributions of the surface magnetic fields and the initial periods of the new born pulsars. The following models are:

1. the surface magnetic fields of the pulsars are described by the distribution derived by Narayan (1987),

$\mathrm{d} N / \mathrm{d}(\log B) \approx 0.065 /\left(B\left[10^{12} G\right]\right)$ year $^{-1}$,

for $B>2 \times 10^{12} \mathrm{G}$. The distribution of pulsars with the surface magnetic fields below $2 \times 10^{12} \mathrm{G}$ bases in Fig. 13 in Narayan (1987). All pulsars are born with the fixed initial period $P_{0}=40 \mathrm{~ms}$ (van der Swaluw \& Wu 2001);

2. the surface magnetic fields of the pulsars as in model (i). The initial pulsar periods are correlated with their surface magnetic fields,

$P_{0}[\mathrm{~ms}]=63.7 /\left(B\left[10^{12} G\right]\right)$,

as postulated by Xu et al. (2001). We apply this formula for the pulsar initial periods above $2 \mathrm{~ms}$; 

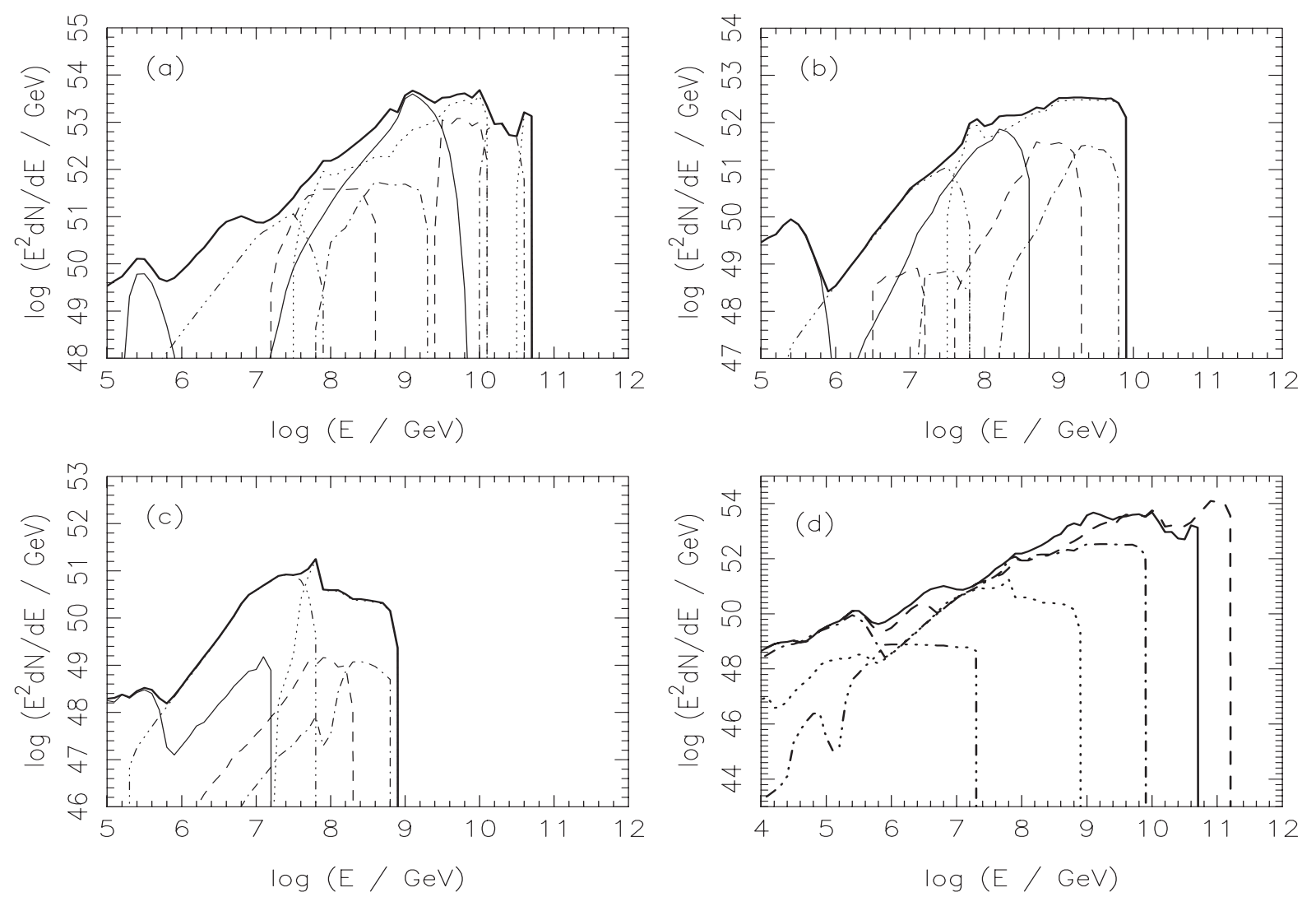

Fig. 1. Energy spectra of different types of nuclei escaping from a supernova nebula into the galactic medium. The nuclei are accelerated by the pulsar with the surface magnetic field $B=4 \times 10^{12} \mathrm{G}$ and different initial periods a) $1 \mathrm{~ms}$, b) $10 \mathrm{~ms}$, and c) $100 \mathrm{~ms}$. The pulsar has been born during the supernova explosion with the envelope mass of $4 M_{\odot}$ expanding with the initial velocity $2000 \mathrm{~km} \mathrm{~s}^{-1}$. The total spectrum of nuclei is marked by the thick full curve. Specific curves show the spectra of nuclei with the mass numbers $A=56$ (dot-dot-dot-dashed), $41-55$ (dotted), 11-40 (dot-dashed), 2-10 (dashed), protons and protons from decay of neutrons (thin full). The spectra of nuclei for pulsars with different initial periods $1 \mathrm{~ms}$ (full curve), $10 \mathrm{~ms}$ (dot-dashed), $10^{2} \mathrm{~ms}$ (dotted), and $10^{3} \mathrm{~ms}$ (dot-dot-dot-dashed) are shown in d). The case of the pulsar with $1 \mathrm{~ms}$ initial period and the expansion velocity of $10^{4} \mathrm{~km} \mathrm{~s}^{-1}$ is shown by the dashed curve.

3. model A of Lorimer et al. (1993) postulating the Gaussian distribution of $\log B$ of the pulsars with parameters $\langle\log B[G]\rangle=12.46$ and $\sigma_{\log B}=0.31$. We apply this model for the range of $B=10^{11.5-13.3} \mathrm{G}$. All pulsars are born with a single initial period, $\log P_{0}[\mathrm{~ms}]=1.35$;

4. model B of Lorimer et al. (1993) postulating the Gaussian distributions of $\log B$, with $\langle\log B[G]\rangle=12.3$ and $\sigma_{\log B}=$ 0.35 , and for $\log P_{0}$, with $\left\langle\log P_{0}[\mathrm{~ms}]\right\rangle=2.6$ and $\sigma_{\log B}=$ 0.35 above $2 \mathrm{~ms}$.

For these four models of the pulsar population, we calculate the injection spectra of nuclei into the Galaxy applying the values of the normalization coefficients, $\xi \cdot \eta$, which give us the flux of calculated nuclei on the level comparable to that observed in the cosmic ray spectrum. It is assumed that the mass of nebula surrounding the pulsar is equal to $3 M_{\odot}$ and expends with the velocity of $2000 \mathrm{~km} \mathrm{~s}^{-1}$. Model (i), calculated for $\xi \cdot \eta=1 / 700 \mathrm{yr}^{-1}$, gives a power law spectrum of nuclei over about two decades above $\sim 10^{8} \mathrm{GeV}$ and very sharp cut-off at lower energies (see Fig. 2i). The spectrum is dominated by heavy nuclei from the iron group. Model (ii), calculated also for $\xi \cdot \eta=1 / 700 \mathrm{yr}^{-1}$, gives very flat spectrum at high energies with the spectral index close to 2 (see Fig. 2ii). The primary iron nuclei disintegrate significantly in this model. Protons from decay of neutrons can contribute to the total spectrum at around $10^{18} \mathrm{eV}$. These features are due to a relatively large number of pulsars with short periods predicted by this model. Model (iii), calculated for $\xi \cdot \eta=1 / 120 \mathrm{yr}^{-1}$, gives the total spectrum with a rather narrow, strong peak at $\sim 10^{8}-10^{9} \mathrm{GeV}$ (see Fig. 2iii). The total spectrum is dominated by heavy nuclei from the iron group. Such shape of the spectrum is due to the assumption about the fixed period of all new born pulsars which is probably not very realistic. Model (iv), $\xi \cdot \eta=1 / 120 \mathrm{yr}^{-1}$, predicts the power law spectrum of nuclei between a few $10^{7} \mathrm{GeV}$ and $10^{10} \mathrm{GeV}$ with the index close to 3 (see Fig. 2iv). The spectrum is dominated by heavy nuclei in all this energy range. In the next section we compare the spectra of nuclei calculated in terms of these models with the observed spectrum and the mass composition of CRs.

\section{Contribution to the cosmic rays in the Galaxy}

Having in hand the injection spectrum of nuclei by pulsars, we can estimate the flux of these nuclei inside the Galaxy adopting the leaky box model from the relation

$\frac{\mathrm{d} N}{\mathrm{~d} E \mathrm{~d} S \mathrm{~d} t \mathrm{~d} \Omega}=\frac{\mathrm{d} N_{\text {inj }}}{\mathrm{d} E \mathrm{~d} t} \cdot \frac{c \tau_{\text {esc }}}{4 \pi V_{\text {gal }}}$, 

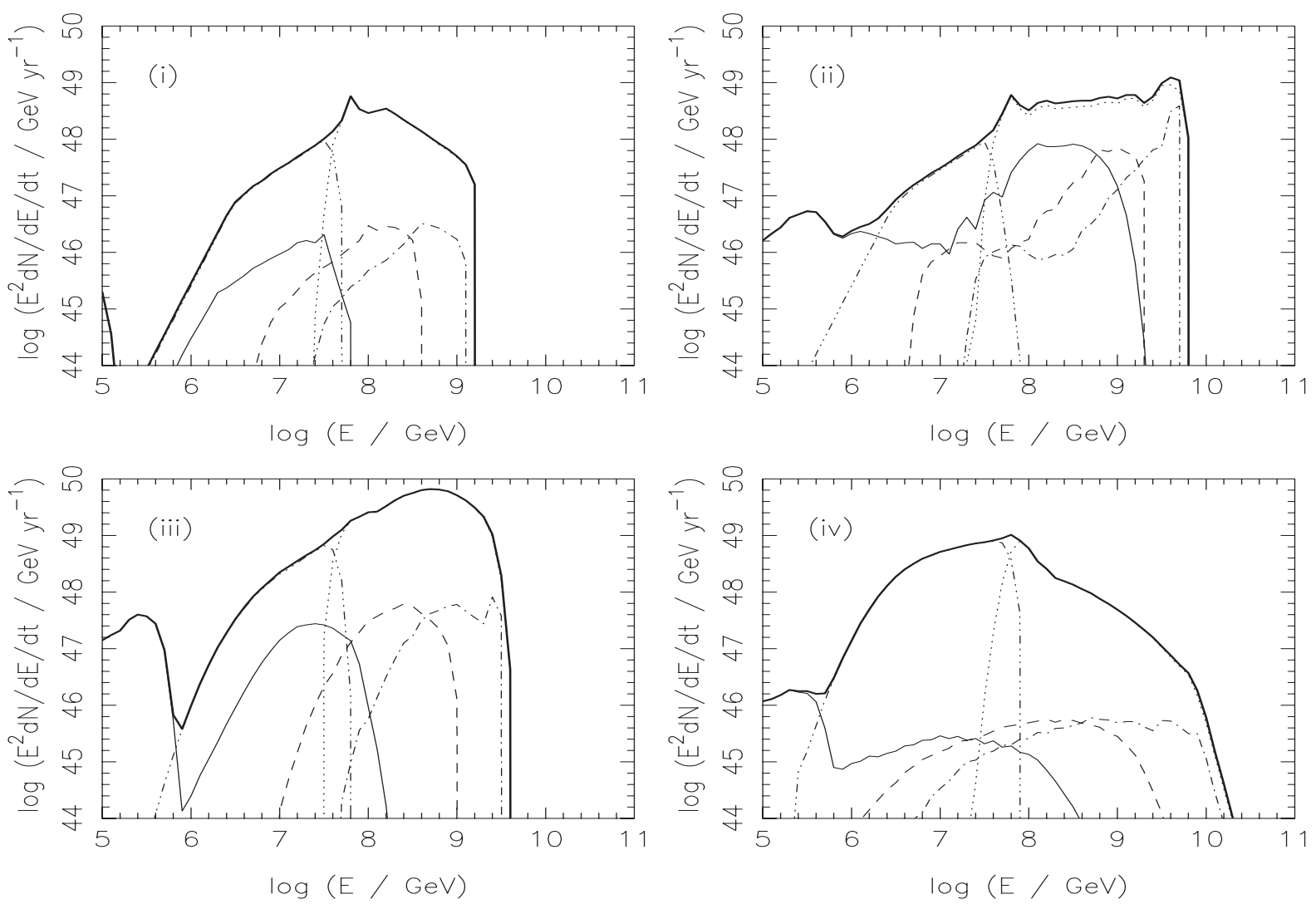

Fig. 2. The spectra of nuclei, injected by the galactic population of pulsars into the Galaxy, calculated for different models of initial parameters of the pulsar population, obtained in the work by Narayan (1987) and van der Swaluw \& Wu (2001) (model i), Narayan (1987) and Xu et al. (2001) (model ii), Model A of Lorimer et al. (1993) (our model iii), and Model B of Lorimer et al. (1993) (model iv) (see text for details). The spectra of different types of nuclei are marked as in Figs. 1.

where $\mathrm{d} N_{\text {inj }} / \mathrm{d} E \mathrm{~d} t$ is the total injection spectrum of CRs from all the pulsars in the Galaxy (calculated in Sect. 2), $V_{\text {gal }}=$ $10^{68} \mathrm{~cm}^{3}$ is the volume of the Galaxy (a disk with a radius of $15 \mathrm{kpc}$ and a half-thickness of $3 \mathrm{kpc}$ ), and $\tau_{\text {esc }}$ is the escape time of hadrons from the Galaxy. The dependence of the escape time of nuclei on their energy and charge is approximated by

$\tau_{\mathrm{esc}}=\frac{2 \times 10^{7} \mathrm{yr}}{\left(E_{\mathrm{GeV}} / Z\right)^{0.3}}$,

where $Z$ is the charge of nuclei and $E_{\mathrm{GeV}}$ its energy in GeV. This formula is normalized to the estimated lifetime of protons with energies $10^{18} \mathrm{eV}$ equal to $4 \times 10^{4}$ years (see Fig. 4.18 in Berezinsky et al. 1990).

The calculations have been performed for all four models of the pulsar population, applying the normalization parameter, $\xi \cdot \eta$, as reported above. Our aim is to find out if any of the proposed so different pulsar population models is able to describe general features of the observed cosmic ray spectrum. The comparison with the CR and calculated spectra are shown in Figs. 3. When fitting the observed CR spectrum in the broad energy range, we assume the popular hypothesis suggesting that the shape of the observed cosmic ray spectrum is a combination of four different components. Below the knee the power law spectrum has the form $\propto E^{-2.7}$. It is widely believed that the main contribution to this part of the spectrum comes from the particles accelerated in the supernova shock waves resulting in a power law spectrum and the exponential cut-off which we put at $10^{7} \mathrm{GeV}, \mathrm{d} N / \mathrm{d} E \propto E^{-2.7} \exp \left(-E / 10^{7} \mathrm{GeV}\right)$. Between the knee and the second knee at a few $10^{17} \mathrm{eV}$, the spectrum has the form $E^{-3}$, and between the second knee and the ankle $\propto E^{-3.2}$. The extremely high energy part of the cosmic ray spectrum is due to another component approximated by a simple power law with the spectral index -2.7 . Particles, accelerated by the pulsars, dominate the spectrum between the knee and the ankle.

However, only model (iv) gives good general consistency of the observed shape of the spectrum with the calculated one. Model (i) predicts significant deficit of particles at $\sim 10^{16} \mathrm{eV}$, which has not been reported by experimentalists. This deficit might be suppressed if the pulsars are able to accelerate nuclei for longer time than the radio activity period expected from the modeling of magnetospheric high energy cascade processes. Models (ii) and (iii) predict too flat spectra between the knee and the ankle and thus should be rejected.

We also calculate the expected mass composition of the CRs, $\langle\ln A\rangle$, in terms of the model (iv) and compare it with the measurements of the mass composition of CRs by different experiments (see Fig. 4). The mass composition of the galactic supernova component is taken as reported by the direct measurements at low energies (JACEE, RUNJOB). The same composition is also applied to the extremely high energy component. We calculate the average mean $\langle\ln A\rangle$ for our model (iv) 

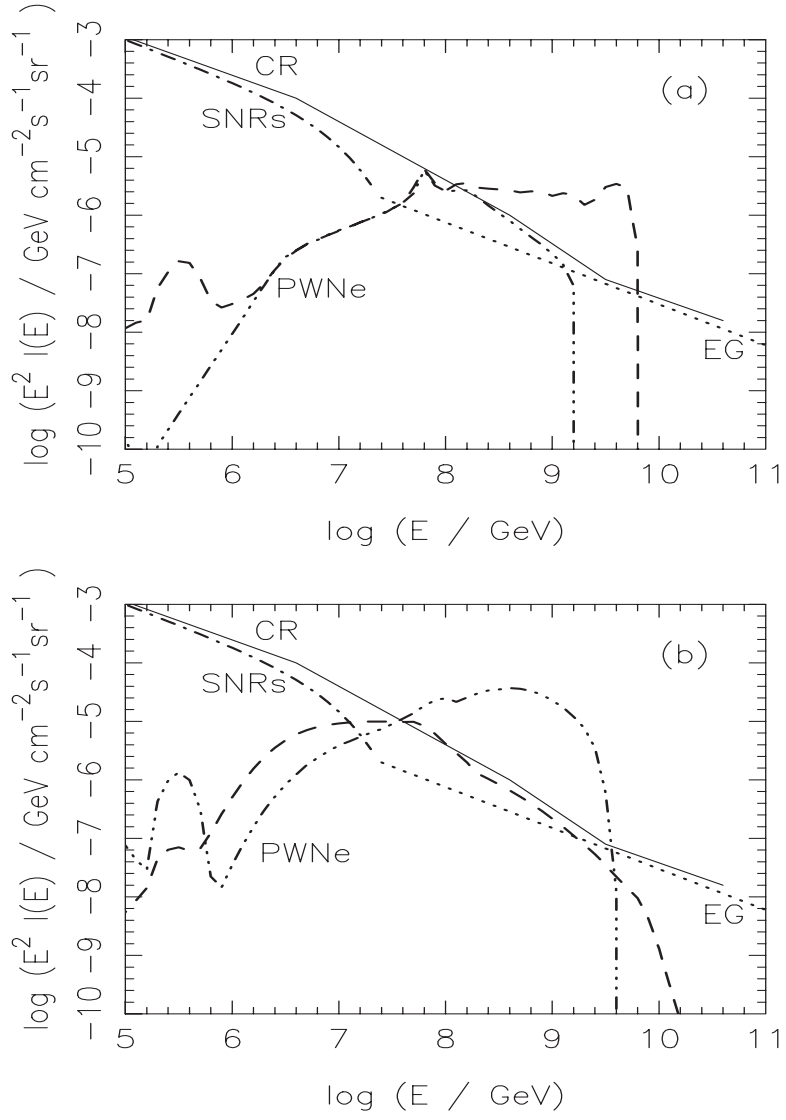

Fig. 3. The comparison of the spectrum of particles accelerated by pulsars with the parameters described by the model (i) (dot-dot-dot-dashed curve in a)), model (ii) (dashed curve in a)), model (iii) (dot-dot-dot-dashed curve in b)), and model (iv) (dashed curve in b)), with the observed cosmic ray spectrum (thin full curve). It is assumed that at low energies the supernova remnants (SNR) accelerate CRs with the spectrum $\mathrm{d} N / \mathrm{d} E \propto E^{-2.7} \exp \left(-E / 10^{7} \mathrm{GeV}\right.$ ) (dotdashed curve). At extremely high energies (EG), the spectrum has the form $\mathrm{d} N / \mathrm{d} E \propto E^{-2.7}$ (dotted line).

assuming two models for extend of the extragalactic (EG) component. In the first one, EG component extends down to at least $3 \times 10^{7} \mathrm{GeV}$. In the second one, it breaks at $10^{9} \mathrm{GeV}$ (see Fig. 4). The Model (iv) with EG component extending to low energies describes well the general tendency reported by most experiments, i.e. the rise of $\langle\ln A\rangle$ above the knee, and gradual decrease of $\langle\ln A\rangle$ above $\sim 3 \times 10^{17} \mathrm{eV}$. Therefore, we conclude that the model of the pulsar contribution to the cosmic ray spectrum can explain the change of the average mass above the knee, which is the consequence of a sudden switching off the pulsar acceleration mechanism of heavy nuclei due to the lack of their extraction from the neutron star surface. In our model this is due to the switching off the process of electromagnetic cascading in the inner pulsar magnetosphere whose products heat up the polar regions of the neutron star. If the light extragalactic component does not extend to low energies but breaks at $\sim 10^{18} \mathrm{eV}$, then our model predicts rather heavy composition up to $\sim 10^{18} \mathrm{eV}$ as suggested by some groups (Dawson et al. 1998; Ave et al. 2003; Dova et al. 2003).

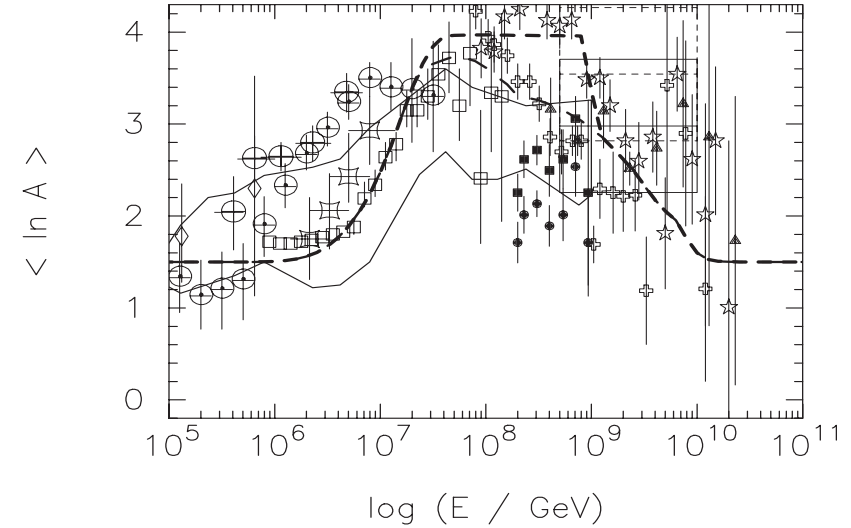

Fig. 4. The comparison of the mass composition of the cosmic rays, $\langle\ln A\rangle$, obtained in terms of the model (iv) for the case of the light extragalactic component extending to low energies (thick dashed curve) and breaking at $10^{18} \mathrm{eV}$ (thin dashed curve), with the measurements reported by different experiments (JACEE - Shibata et al. 1999, RUNJOB - Apanasenko et al. 2001, CASA-MIA Glasmacher et al. 1999, KASCADE (e/m) - Ulrich et al. 2001, $\operatorname{KASCADE}(\mathrm{p} / \mathrm{m})$ - Engler et al. 1999, EAS-TOP - Alessandro et al. 2001, FLY'S-EYE - Dawson et al. 1998, Akeno A1 - Hayashida et al. 1995, Akeno A100 - Hayashida et al. 1997, Haverah Park - Ave et al. 2003, Volcano Ranch - Dova et al. 2003). The region between the thin full curves shows $\langle\ln A\rangle$ obtained from many experiments and derived by Hörandel (2004). $\langle\ln A\rangle$ equal to 1.5 is taken for the SNR contribution to cosmic ray spectrum as measured at low energies by direct experiments. Similar composition is also applied for the extragalactic (EG) component of CRs at the highest energies.

\section{Discussion and conclusions}

The best fit to the observed cosmic ray spectrum between the knee and the ankle is obtained for the model B of Lorimer et al. (1993) (our model iv) which postulates that the observed radio pulsars are born with relatively long initial periods, with the average value of $\sim 400 \mathrm{~ms}$, with the Gaussian distribution in $\log$ scale, and typical surface magnetic fields, with the average value of $2 \times 10^{12} \mathrm{G}$ and also the Gaussian distribution in $\log$ scale. This average value of the pulsar periods at birth is similar to that one obtained in the analytical work by Giller \& Lipski (2002), who got the best description of the cosmic ray spectrum for the gamma distribution of the initial pulsar periods (which is $\propto P_{0}^{s-1}$ for small periods) with average value of $P_{0}=500 \mathrm{~ms}$ and $s=3.86$, and the Gaussian distribution for $\log B$ with average value between $10^{12}-10^{13} \mathrm{G}$ and $\sigma_{\log B}=0.4$. However, the shape of the pulsar distribution for initial periods is very different from that one derived by Lorimer et al. (1993), and applied in our work. This is not surprising since, in contrast to Giller \& Lipski (2002), we take into account the propagation and escape conditions, and adiabatic and collisional energy losses of nuclei during their passage throughout the pulsar wind nebula. These processes have strong effects on the injection spectrum of nuclei into the Galaxy. From the normalization of the calculated spectrum of nuclei in terms of the model (iv) to the observed cosmic ray spectrum, we obtained the efficiency of conversion of the rotational energy of the pulsar into the energy of iron nuclei multiplied by the pulsar birth rate equal to $\sim 1 / 120 \mathrm{yr}^{-1}$. This value is consistent with the estimated 
pulsar birth rate $\sim 1 /(20-250 \mathrm{yr}$ ) (Lyne et al. 1985; Lorimer 2003; Vranesevic et al. 2003). Therefore, we conclude that the efficiency of acceleration of the iron nuclei should be in the range $\sim(0.2-1.0)$.

The low energy break in the spectrum of nuclei injected by the PWNe is explained in our model by switching off the mechanism of extraction of the iron nuclei from the surface of the neutron star. This is caused by the lack of efficient cascading in the inner pulsar magnetosphere which products impinge on the region of the polar cap and allow efficient extraction of the iron nuclei from the neutron star surface. The cascades stop developing in the pulsar magnetosphere when the pulsar period becomes too low due to the pulsar rotational energy losses. This happens when pulsars reach the age of $\sim 10^{6}-10^{7} \mathrm{yr}$, depending on the value of their surface magnetic field. Note however, that Giller \& Lipski (2002) explain this low energy cut-off by the decay of the surface magnetic field of the pulsars on a typical time scale of $\sim 10^{7} \mathrm{yr}$.

The models (i) and (iii) for the initial parameters of the pulsar population are not consistent with the observed spectral shape of the CRs, since they give too narrow spectra with the peak somewhere between the knee and the ankle. Model (ii), postulating a correlation between the initial pulsar period and the strength of the surface magnetic field of the the pulsar $(\mathrm{Xu}$ et al. 2001), overproduce CRs in the region of the ankle by about an order of magnitude, even for the assumed here relatively strong dependence of the lifetime of particles inside the Galaxy on their energy.

The numerical calculations presented here allow us to include the energy loss processes of nuclei, accelerated by the pulsar, during their propagation inside the PWNe and also consider their escape conditions from the PWNe. Therefore, we can predict the mass composition of nuclei injected from the PWNe into the Galaxy and compare it with the observations. The mass composition estimated in terms of our best fit model (iv) shows rise in the energy range $\sim 10^{6}-10^{7} \mathrm{GeV}$, and slower fall between $\sim 10^{8} \mathrm{GeV}$ and a few $10^{9} \mathrm{GeV}$, provided that the light extragalactic component expected at the highest energies extends down to $3 \times 10^{7} \mathrm{GeV}$. This behaviour of the mass composition is in general agreement with the most observational results available in the literature and collected in the paper by Hörandel (2004) (see Fig. 4). However, if the extragalactic component breaks at $10^{9} \mathrm{GeV}$, then our model predicts heavy composition below $10^{9} \mathrm{GeV}$ as suggested by some other experiments (Dawson et al. 1998; Ave et al. 2003; Dova et al. 2003).

The models considered by us have also other interesting, worth to be mentioned features. For example, model (ii), postulating that a significant part of pulsars are born with short initial periods and strong surface magnetic fields (correlation between these two parameters), predicts domination of the mass composition at energy $\sim 10^{9} \mathrm{GeV}$ by protons from decay of neutrons released from nuclei inside the PWNa. Note that the existence of an additional pulsar population inside the Galaxy with extreme parameters seems to be required by the observations of anomalous X-ray pulsars and magnetars. It can be responsible for the observed anisotropies of CRs from directions of the Galactic Centre and the Cygnus region (see Bednarek 2002;
Bednarek 2003). This pulsar population might be also responsible for the highest energy component of the CR spectrum $\left(>10^{19} \mathrm{eV}\right)$, provided that heavy nuclei are able to escape from the PWNe (see Blasi et al. 2000; and Fig. 1d in this paper). Such model predicts very heavy composition of the highest energy CRs.

It might be surprising that we postulate a similar order contribution to the cosmic ray spectrum around the knee region from particles accelerated at the outer supernova shock waves and from the pulsars. However, it is likely that the initial parameters of pulsars and expanding supernova envelopes, which originate in this same phenomenon, are in some way related. Pulsars with more extreme parameters (initial periods, surface magnetic fields) seem to be produced by explosions of type $\mathrm{Ib} / \mathrm{c}$ supernovae which progenitors rotate fast and have relatively smaller envelopes just before explosion. Therefore, their envelopes have larger velocities, expanding to larger distances and producing outer shocks with larger dimensions than in the case of more massive type II supernovae. Such shocks allow more efficient particle acceleration. Moreover, the exact shape of the cosmic ray spectrum in the knee region is not precisely known leaving place for some speculations concerning its smoothness. For example, Erlykin \& Wolfendale (1997) argue for the existence of fine structures in the knee region of the cosmic ray spectrum to be interpreted as contribution from nuclei with different mass numbers.

The rate of pulsar formation inside the Galaxy, estimated on 1/(20-250 yr) (Lyne et al. 1985; Lorimer 2003; Vranesevic et al. 2003), is about three orders of magnitude higher than the GRB event rate in our Galaxy. These pulsars can supply hadrons with the rate required by the observed density of the CRs in the Galaxy. Therefore, the population of classical radio pulsars observed in the Galaxy can provide at least similar contribution to the galactic CRs as recent GRB model considered by Wick et al. (2004).

Acknowledgements. We would like to thank M. Giller and the anonymous referee for many useful comments. This work is supported by the Polish KBN grants No. 5P03D 02521 and PBZ-KBN-054/P03/2001.

\section{References}

Abu-Zayyad, T., Belov, K., Bird, D. J., et al. 2001, ApJ, 557, 686

Alessandro, B., for the EAS-TOP collaboration 2001, in Proc. 27th ICRC, Hamburg, 1, 124

Apanasenko, A. V., Sukhadolskaya, V. A., Debrina, V. A., et al. 2001, $\mathrm{APh}, 16,13$

Arons, J. 1998, in Elba Conf. on Pulsars and their Nebulae, Mem. Soc. Ast. Ital., 69, 989

Arons, J. 2003, ApJ, 589, 871

Arqueros, F., Barrio, J. A., Bernlohr, K., et al. 2000, A\&A, 359, 682

Atoyan, A. M., \& Aharonian, F. A. 1996, MNRAS, 278, 525

Ave, M., Cazon, L., Hinton, J. A., et al. 2003, APh, 19, 61

Bednarek, W. 2002, MNRAS, 331, 483

Bednarek, W. 2003, MNRAS, 345, 847

Bednarek, W., \& Bartosik, M. 2003, A\&A, 405, 689

Bednarek, W., \& Protheroe, R. J. 1997, PRL, 79, 2616

Bednarek, W., \& Protheroe, R. J. 2002, APh, 16, 397

Bell, A. R., \& Lucek, S. G. 2001, MNRAS, 321, 433 
Bellido, J. A., Clay, R. W., Dawson, B. R., \& Johnston-Hollitt, M. 2001, APh, 15, 167

Berezinsky, V. S., Bulanov, S. V., Dogiel, V. A., \& Ptuskin, V. S. 1990, Astrophysics of cosmic rays, North-Holland, Amsterdam

Biermann, P. L. 1993, A\&A, 271, 649

Bird, D. J., Corbato, S. C., Dai, H. Y., et al. 1993, PRL, 71, 3401

Blasi, P., Epstein, R. I., \& Olinto, A. V. 2000, ApJ, 533, L123

Burnett, T. H., Dake, S., Derrickson, J. H., et al. 1990, ApJ, 349, 25

Bykov, A. M. 2001, SSRv, 99, 317

Cesarsky, C. J., \& Montmerle, T. 1983, SSRv, 36, 173

Cheng, K. S., \& Chi, X. 1996, A\&A, 306, 326

Cheng, K. S., Ho, C., \& Ruderman, M. 1986, ApJ, 300, 500

Davidson, K., \& Fesen, R. A. 1985, ARA\&A, 23, 119

Dawson, B. R., Meyhandan, R., \& Simpson, K. M. 1998, APh, 9, 331

Dova, M. T., Mancenido, M. E., Mariazzi, A. G., McCauley, T. P., \& Watson, A. A. 2003, APh, submitted [arXiv: astro-ph/0312463]

Drury, L. O'C., van der Swaluw, E., \& Carroll, O. 2003 [arXiv: astro-ph/0309820]

Engler, J., Antoni, T., Apel, W. D., et al. 1999, in Proc. 26th ICRC, Salt Lake City, 1, 349

Erlykin, A. D., \& Wolfendale, A. W. 1997, J. Phys. G, 23, 979

Fesen, R. A., Shull, J. M., \& Hurford, A. P. 1997, AJ, 113, 354

Fowler, J. W., Fortson, L. F., Jui, C. C., et al. 2001, APh, 15, 49

Gallant, Y. A., \& Arons, J. 1994, ApJ, 435, 230

Gil, J., Melikidze, G. I., \& Geppert, U. 2003, A\&A, 407, 315

Giller, M., \& Lipski, M. 2002, J. Phys. G, 28, 1275

Glasmacher, M. A. K., Catanese, M. A., Chantell, M. C., et al. 1999, APh, 10, 291

Hayashida, N., Honda, K., Honda, M., et al. 1995, J. Phys. G, 21, 1101

Hayashida, N., Honda, K., Honda, M., et al. 1997, in Proc. 25th ICRC (Durban), 6, 241

Hayashida, N., Nagano, M., Nishikawa, D., et al. 1999, APh, 10, 303 Helfand, D. J., Gotthelf, E. V., \& Halpern, J. P. 2001, ApJ, 556, 380

Ho, C. 1989, ApJ, 342, 396

Hoshino, M., Arons, J., Gallant, Y. A., \& Langdon, A. B. 1992, ApJ, 390,454

Hörandel, J. R. 2003, APh, 19, 193

Hörandel, J. R. 2004, APh, in press [arXiv : astro-ph/0402356]
Jokipii, J. R., \& Morfill, G. E. 1985, ApJ, 290, L1

Karakuła, S., Osborne, J. L., \& Wdowczyk, J. 1974, J. Phys. G, 7, 437

Kennel, C. F., \& Coroniti, F. V. 1984, ApJ, 283, 694

Lorimer, D. R., Bailes, M., Dewey, R. J., \& Harrison, P. A. 1993, MNRAS, 263, 403

Lorimer, D. R. 2003, in Proc. Young Neutron Stars and Their Environments, ed. F. Camilo, \& B. M. Gaensler, IAU Symp., v.218 [arXiv: astro-ph/0308501]

Lucek, S. G., \& Bell, A. R. 2000, MNRAS, 314, 65

Lyne, A. G., Manchester, R. N., \& Taylor, J. H. 1985, MNRAS, 213, 613

Maurin, D., Casse, M., \& Vangioni-Flam, E. 2003, APh, 18, 471

Narayan, R. 1987, ApJ, 319, 162

Ostriker, J. P., \& Gunn, J. E. 1969, ApJ, 157, 1395

Ostriker, J. P., \& Gunn, J. E. 1971, ApJ, 164, L95

Peters, B. 1961, Nuovo Cimento, 22, 800

Protheore, R. J., \& Szabo, A. P. 1992, PRL, 69, 2885

Rees, M. J., \& Gunn, J. E. 1974, MNRAS, 167, 1

Roth, M., et al. (KASCADE Collaboration) 2003, Proc. 28th Int. Cosmic Ray Conf., Tsukuba, Japan, 1, 139

Ruderman, M., \& Cheng, K. S. 1988, ApJ, 157, 869

Shibata, T. 1999, Nucl. Phys. B Suppl., 75, 22

Shibata, S., Tomatsuri, H., Shimanuki, M., Saito, K., \& Mori, K. 2003, MNRAS, 346, 841

Shirasaki, Y., Kakimoto, F., Ogio, S., et al. 2001, APh, 15, 357

Stanev, T., Biermann, P. L., \& Gaisser, T. K. 1993, A\&A, 274, 902

Ulrich, H., Antoni, T., Apel, W. D., et al. 2001, in Proc. 27th Int. Cosmic Ray Conf., Hamburg, 1, 97

van der Swaluw, E., \& Wu, Y. 2001, ApJ, 555, L49

Vietri, M. 1995, ApJ, 453, 883

Vranesevic, N., Manchester, R. N., Hobbs, G. B., et al. 2003, in Proc. Young Neutron Stars and Their Environments, ed. F. Camilo, \& B. M. Gaensler, IAU Symp., v.218 [arXiv: astro-ph/0310201] Waxman, E. 1995, PRL, 75, 386

Wick, S. D., Dermer, C. D., \& Atoyan, A. 2004, APh, in press [arXiv:astro-ph/0310667]

Xu, R. X., Wang, H. G., \& Qiao, G. J. 2001, Chin. J. A\&A, 2, 533 [arXiv: astro-ph/0206166]

Yoshida, S., Hayashida, N., Honda, K., et al. 1995, APh, 3, 105 